PROCEEDINGS OF THE

AMERICAN MATHEMATICAL SOCIETY

Volume 135, Number 12, December 2007, Pages 3895-3903

S 0002-9939(07)08939-3

Article electronically published on September 10, 2007

\title{
HOMOGENEOUS POLYNOMIALS ON STRICTLY CONVEX DOMAINS
}

\author{
PIOTR KOT
}

(Communicated by Mei-Chi Shaw)

\begin{abstract}
We consider a circular, bounded, strictly convex domain $\Omega \subset \mathbb{C}^{d}$ with boundary of class $C^{2}$. For any compact subset $K$ of $\partial \Omega$ we construct a sequence of homogeneous polynomials on $\Omega$ which are big at each point of $K$. As an application for any $E \subset \partial \Omega$ circular subset of type $G_{\delta}$ we construct a holomorphic function $f$ which is square integrable on $\Omega \backslash \mathbb{D} E$ and such that $E=E_{\Omega}^{2}(f):=\left\{z \in \partial \Omega: \int_{\mathbb{D} z}|f|^{2} d \mathfrak{L}_{\mathbb{D} z}^{2}=\infty\right\}$ where $\mathbb{D}$ denotes unit disc in $\mathbb{C}$.
\end{abstract}

\section{INTRODUCTION}

Let $\Omega$ denote a bounded, convex and circular domain with a defining function $\eta$ of class $C^{2}$. We also denote by $\mathbb{D}$ the unit disc in $\mathbb{C}$ and define the exceptional set $E_{\Omega}^{2}(f):=\left\{z \in \partial \Omega: \int_{\mathbb{D} z}|f|^{2} d \mathfrak{L}_{\mathbb{D} z}^{2}=\infty\right\}$ for a holomorphic function $f \in \mathbb{O}(\Omega)$. For more information about exceptional sets see [1, 2, 3, 4, 5, 6, 17.

In the paper [12] a natural number $K$ and a sequence $\left\{p_{n}\right\}_{n=0}^{\infty}$ of homogeneous polynomials on $\mathbb{C}^{d}$ were constructed so that $\left|p_{n}(z)\right| \leq 2$ and $\sum_{j=K m}^{K(m+1)-1}\left|p_{n}(z)\right| \geq$ 0.5 for all $z$ belonging to the boundary of the unit ball $\partial \mathbb{B}^{d}$. In the paper [7] we introduced some additional arguments in such a way that for any circular set $E \subset \partial \mathbb{B}^{d}$ of type $G_{\delta}$ and $F_{\sigma}$ we could construct a holomorphic function $f$ on the unit ball $\mathbb{B}^{d}$ so that $E_{\mathbb{B}^{d}}^{2}(f)=E$.

In this paper we construct similar homogeneous polynomials as in [7, 10, 11, 12]. Whilst these papers dealt with homogeneous polynomials on the unit ball, in this paper we construct homogeneous polynomials on $\Omega$ which is a bounded, circular and strictly convex domain with boundary of class $C^{2}$.

1.1. Geometric notions. In the complex $d$-dimensional space $\mathbb{C}^{d}$ we consider the natural scalar product $\langle\circ, \circ\rangle$. We also consider rotation invariant pseudometrics

$$
\rho(z, w)=\min _{|\lambda|=1}\|z-\lambda w\| .
$$

As usual, by $B(\xi ; r)$ we denote the open ball with center $\xi$ and radius $r$, i.e.

$$
B(\xi ; r):=\left\{z \in \mathbb{C}^{d}: \rho(\xi, z)<r\right\}
$$

Received by the editors September 8, 2005 and, in revised form, September 20, 2006.

2000 Mathematics Subject Classification. Primary 32A05, 32A40.

Key words and phrases. homogeneous polynomials, exceptional sets, highly nonintegrable holomorphic function. 
We claim that there exist constants $0<q_{0}<q_{1}$ such that

$$
q_{0} r^{2 d-1} \leq \mathfrak{L}^{2 d}(B(\xi ; r)) \leq q_{1} q_{0} r^{2 d-1}
$$

for $\xi \in \partial \Omega$ and $0 \leq r \leq 2 R:=2 \sup _{z, w \in \partial \Omega} \rho(z, w)$.

Since rotation does not change $\mathfrak{L}^{2 d}(B(\xi ; r))$ we can assume that $\xi=(a, 0)$ for some $a \in \mathbb{R}_{+}$. In particular we can calculate

$$
\rho((\lambda, w),(a, 0))=\min _{|\eta|=1}\|(\eta \lambda, \eta w)-(a, 0)\|=\sqrt{(|\lambda|-a)^{2}+\|w\|^{2}} .
$$

Assume for a moment that $r \leq a$. Since $\xi=(a, 0)$ we can observe that $B(\xi ; r)=$ $B_{-}(\xi ; r) \cup B_{+}(\xi ; r)$ where:

$$
\begin{aligned}
& B_{+}(\xi ; r):=\left\{\left((a+s) e^{i \phi}, w\right) \in \mathbb{C}^{d}: 0 \leq s<r, \phi \in[0,2 \pi],\|w\|<\sqrt{r^{2}-s^{2}}\right\} \\
& B_{-}(\xi ; r):=\left\{\left((a-s) e^{i \phi}, w\right) \in \mathbb{C}^{d}: 0<s<r, \phi \in[0,2 \pi],\|w\|<\sqrt{r^{2}-s^{2}}\right\} .
\end{aligned}
$$

We may calculate1

$$
\begin{aligned}
\mathfrak{L}^{2 d}(B(\xi ; r)) & =\int_{B_{+}(\xi ; r)} 1 d \mathfrak{L}^{2 d}+\int_{B_{-}(\xi ; r)} 1 d \mathfrak{L}^{2 d} \\
& =\int_{0}^{2 \pi} \int_{0}^{r} \int_{\|w\|<\sqrt{r^{2}-s^{2}}}(a+s)+(a-s) d \mathfrak{L}^{2 d-2}(w) d s d \phi \\
& =4 \pi a \pi_{2 d-2} \int_{0}^{r}\left(r^{2}-s^{2}\right)^{d-1} d s \\
& =4 \pi a \pi_{2 d-2} \int_{0}^{r} \sum_{k=0}^{d-1}\left(\begin{array}{c}
d-1 \\
k
\end{array}\right)(-1)^{k} r^{2(d-1-k)} s^{2 k} d s \\
& =4 \pi a \pi_{2 d-2} \sum_{k=0}^{d-1}\left(\begin{array}{c}
d-1 \\
k
\end{array}\right) \frac{(-1)^{k}}{2 k+1} r^{2 d-1} .
\end{aligned}
$$

Since $0<r<2 R$ and $0 \in \Omega$ the above equality implies that there exist constants $0<q_{0}<q_{1}$ such that (11) holds. Let us observe that if $r \leq \min _{\xi \in \partial \Omega}\|\xi\|$ we have $\mathfrak{L}^{2 d}(B(\xi ; r))=r^{2 d-1}\|\xi\| \sum_{k=0}^{d-1} \frac{(-1)^{k} 4 \pi \pi_{2 d-2}(d-1) !}{(2 k+1) k !(d-1-k) !}$ for $\xi \in \partial \Omega$. However for us it suffices to use (1).

Additionally let us assume that $\mathfrak{L}^{2 d}(B(0 ; 2 R)) \leq q_{1} q_{0}$.

A subset $A \subset \mathbb{C}^{d}$ is called $\alpha$-separated if $\rho\left(z_{1}, z_{2}\right)>\alpha$ for all distinct elements $z_{1}$ and $z_{2}$ of $A$. It is clear that for $\alpha>0$ each $\alpha$-separated subset of $\partial \Omega$ is finite.

If $g: \mathbb{C}^{d} \rightarrow \mathbb{C}$ is a function of class $C^{2}$, then we denote $g_{\xi}=\left(\frac{\partial g}{\partial z_{1}}(\xi), \ldots, \frac{\partial g}{\partial z_{d}}(\xi)\right)$ and

$$
H_{g}(P, w):=\frac{1}{2} \sum_{j, k=1}^{d} \frac{\partial^{2} g}{\partial z_{j} \partial z_{k}}(P) w_{j} w_{k}+\frac{1}{2} \sum_{j, k=1}^{d} \frac{\partial^{2} g}{\partial \bar{z}_{j} \partial \bar{z}_{k}}(P) \bar{w}_{j} \bar{w}_{k}+\sum_{j, k=1}^{d} \frac{\partial^{2} g}{\partial z_{j} \partial \bar{z}_{k}}(P) w_{j} \bar{w}_{k} .
$$

Let us recall that $\eta$ is a defining function of class $C^{2}$ for $\Omega$. Let $X$ be a compact, circular set. Assume that $X$ contains only strictly convex points of $\partial \Omega$, i.e. if $\xi \in X$, then $H_{\eta}(\xi, w)>0$ where $w \neq 0$ and $\Re\left(\left\langle w, \overline{\eta_{\xi}}\right\rangle\right)=0$.

\footnotetext{
${ }^{1} \pi_{m}:=\mathfrak{L}^{m}\left(\left\{w \in \mathbb{C}^{m}:\|w\|<1\right\}\right)$.
} 


\section{Homogeneous polynomials}

All homogeneous polynomials of degree $n$ constructed in this paper have the following form:

$$
p_{n}(z)=\sum_{\xi \in A}\left\langle z, \nu_{\xi}\right\rangle^{n}
$$

where $A$ is a finite subset of $\partial \Omega$ and $\nu_{\xi}=\frac{1}{\left\langle\xi, \overline{\eta_{\xi}}\right\rangle} \overline{\eta_{\xi}}$.

We begin with a very important estimation of $\left|\left\langle z, \nu_{\xi}\right\rangle\right|$.

Lemma 2.1. There exist constants $c_{1}, c_{2}>0$ such that

$$
c_{1} \rho^{2}(z, \xi) \leq 1-\left|\left\langle z, \nu_{\xi}\right\rangle\right| \leq c_{2} \rho^{2}(z, \xi)
$$

for $\xi \in X, z \in \partial \Omega$.

Proof. Since $\Omega$ is a circular and convex domain

$$
\left|\left\langle z, \overline{\eta_{\xi}}\right\rangle\right|-\left|\left\langle\xi, \overline{\eta_{\xi}}\right\rangle\right| \leq\left|\left\langle z, \overline{\eta_{\xi}}\right\rangle\right|-\Re\left\langle\xi, \overline{\eta_{\xi}}\right\rangle \leq \max _{|\lambda|=1} \Re\left\langle\lambda z-\xi, \overline{\eta_{\xi}}\right\rangle \leq 0 .
$$

First we prove that for $\xi \in \partial \Omega$ we have the following property:

$$
\left\langle\xi, \overline{\eta_{\xi}}\right\rangle \in \mathbb{R}_{+} \text {. }
$$

Let $\lambda_{0}$ be such that $\left\langle\xi, \lambda_{0} \overline{\eta_{\xi}}\right\rangle \in \mathbb{R}_{+}$and $\left|\lambda_{0}\right|=1$. Observe that

$$
\Re\left\langle z-\xi, \lambda_{0} \overline{\eta_{\xi}}\right\rangle \leq\left|\left\langle z, \overline{\eta_{\xi}}\right\rangle\right|-\left|\left\langle\xi, \overline{\eta_{\xi}}\right\rangle\right| \leq 0
$$

for $z \in \partial \Omega$. Since $\partial \Omega$ is of class $C^{2}$ we have $\overline{\eta_{\xi}}=\lambda_{0} \overline{\eta_{\xi}}$. In particular $\lambda_{0}=1$.

In the next step we prove that there exist constants $c_{3}, c_{4}>0$ such that for $z \in \partial \Omega$ and $\xi \in X$ we have:

$$
c_{3}\|z-\xi\|^{2} \leq\left|\Re\left\langle z-\xi, \overline{\eta_{\xi}}\right\rangle\right| \leq c_{4}\|z-\xi\|^{2} .
$$

Due to [9, Lemma 3.1.6] there exist a defining function $\widetilde{\eta}$ of class $C^{2}$ for $\Omega$ and constants $c_{5}, c_{6}>0$ such that $c_{5}\|w\|^{2} \leq H_{\widetilde{\eta}}(\xi, w) \leq c_{6}\|w\|^{2}$ for $\xi \in X$ and $w \in \mathbb{C}^{d}$. Let

$$
\phi(\xi, h):=\left\langle h, \overline{\widetilde{\eta}_{\xi}}\right\rangle+\overline{\left\langle h, \overline{\widetilde{\eta}_{\xi}}\right\rangle}-\widetilde{\eta}(\xi+h) .
$$

Since $\widetilde{\eta}$ is of class $C^{2}$ we have $\widetilde{\eta}(\xi+h)=\widetilde{\eta}(\xi)+\left\langle h, \overline{\widetilde{\eta}_{\xi}}\right\rangle+\overline{\left\langle h, \overline{\widetilde{\eta}_{\xi}}\right\rangle}+H_{\widetilde{\eta}}(\xi, h)+$ $f(\xi, h)\|h\|^{2}$ where $f$ is a continuous function such that $\lim _{h \rightarrow 0} f(\xi, h)=0$. Observe that $2 \Re\left\langle z-\xi, \widetilde{\widetilde{\eta}_{\xi}}\right\rangle=\phi(\xi, z-\xi)$ for $z \in \partial \Omega$ and $\xi \in X$. In particular we may estimate

$$
\frac{2 \Re\left\langle z-\xi, \overline{\widetilde{\eta}_{\xi}}\right\rangle}{\|z-\xi\|^{2}}=\frac{-H_{\widetilde{\eta}}(\xi, z-\xi)-f(\xi, z-\xi)\|z-\xi\|^{2}}{\|z-\xi\|^{2}} \leq-c_{5}-f(\xi, z-\xi)
$$

and

$$
\frac{2 \Re\left\langle z-\xi, \overline{\widetilde{\eta}_{\xi}}\right\rangle}{\|z-\xi\|^{2}} \geq-c_{6}-f(\xi, z-\xi) .
$$

The above inequalities imply that there exist constants $c_{7}, c_{8}>0$ such that

$$
c_{7}\|z-\xi\|^{2} \leq\left|\Re\left\langle z-\xi, \overline{\widetilde{\eta}_{\xi}}\right\rangle\right| \leq c_{8}\|z-\xi\|^{2} .
$$


Since $\eta, \widetilde{\eta}$ are defining functions for $\Omega$ there exists a continuous, positive function $g$ such that

$$
\left(\frac{\partial \eta}{\partial x_{1}}, \frac{\partial \eta}{\partial y_{1}}, \ldots, \frac{\partial \eta}{\partial x_{d}}, \frac{\partial \eta}{\partial y_{d}}\right)=g(\xi)\left(\frac{\partial \widetilde{\eta}}{\partial x_{1}}, \frac{\partial \widetilde{\eta}}{\partial y_{1}}, \ldots, \frac{\partial \widetilde{\eta}}{\partial x_{d}}, \frac{\partial \widetilde{\eta}}{\partial y_{d}}\right) .
$$

In particular $\Re\left\langle z-\xi, \overline{\eta_{\xi}}\right\rangle=g(\xi) \Re\left\langle z-\xi, \overline{\widetilde{\eta}_{\xi}}\right\rangle$ and there exist constants $c_{3}, c_{4}>0$ such that (5) holds.

Now we prove the main conclusion. Let constants $c_{3}, c_{4}>0$ be such that (50) holds. Let $\lambda_{1}, \lambda_{2}$ be such that $\min _{|\lambda|=1}\left|\Re\left\langle\lambda z-\xi, \overline{\eta_{\xi}}\right\rangle\right|=\left|\Re\left\langle\lambda_{1} z-\xi, \overline{\eta_{\xi}}\right\rangle\right|$ and $\rho(z, \xi)=\min _{|\lambda|=1}\|\lambda z-\xi\|=\left\|\lambda_{2} z-\xi\right\|$. By (5) we may estimate

$$
\begin{aligned}
c_{3}\left\|\lambda_{2} z-\xi\right\|^{2} & \leq c_{3}\left\|\lambda_{1} z-\xi\right\|^{2} \leq\left|\Re\left\langle\lambda_{1} z-\xi, \overline{\eta_{\xi}}\right\rangle\right| \\
& \leq\left|\Re\left\langle\lambda_{2} z-\xi, \overline{\eta_{\xi}}\right\rangle\right| \leq c_{4}\left\|\lambda_{2} z-\xi\right\|^{2} .
\end{aligned}
$$

By (44) we have $\left|\Re\left\langle\lambda_{1} z-\xi, \overline{\eta_{\xi}}\right\rangle\right|=\left\langle\xi, \overline{\eta_{\xi}}\right\rangle-\left|\left\langle z, \overline{\eta_{\xi}}\right\rangle\right|$. In particular we may estimate

$$
\frac{c_{3}}{\left\langle\xi, \overline{\eta_{\xi}}\right\rangle} \rho^{2}(z, \xi) \leq \frac{\left\langle\xi, \overline{\eta_{\xi}}\right\rangle-\left|\left\langle z, \overline{\eta_{\xi}}\right\rangle\right|}{\left\langle\xi, \overline{\eta_{\xi}}\right\rangle}=1-\left|\left\langle z, \nu_{\xi}\right\rangle\right| \leq \frac{c_{4}}{\left\langle\xi, \overline{\eta_{\xi}}\right\rangle} \rho^{2}(z, \xi) .
$$

Since $X$ is a compact set and $\left\langle\xi, \overline{\eta_{\xi}}\right\rangle>0$ it is enough to define $c_{1}=\inf _{\xi \in X} \frac{c_{3}}{\left\langle\xi, \overline{\eta_{\xi}}\right\rangle}$ and $c_{2}=\sup _{\xi \in X} \frac{c_{4}}{\left\langle\xi, \eta_{\xi}\right\rangle}$

In order to control the values of the constructed polynomials we need some information about $\alpha$-separated sets.

Lemma 2.2. Suppose that $A=\left\{\xi_{1}, \ldots, \xi_{s}\right\}$ is a $2 \alpha$-separated subset of $\partial \Omega$. For $z \in \partial \Omega$ let

$$
A_{k}(z):=\{\xi \in A: \alpha k t \leq \rho(z, \xi) \leq \alpha(k+1) t\} .
$$

Then the set $A_{k}(z)$ has at most $q_{1}(k+2)^{2 d-1}$ elements. The set $A_{0}$ has at most 1 element and $s \leq q_{1}(\alpha t)^{1-2 d}$.

Proof. Observe that $B\left(\xi_{1} ; \alpha t\right) \cap B\left(\xi_{1} ; \alpha t\right)=\emptyset$ for $\xi_{1} \neq \xi_{2} \in A$. Moreover

$$
\bigcup_{\xi \in A_{k}(z)} B(\xi ; \alpha t) \subset B(z ; \alpha(k+2) t) .
$$

Let $d_{k}$ be a number of elements in $A_{k}(z)$. In particular

$$
d_{k} q_{0}(\alpha t)^{2 d-1} \leq \sum_{\xi \in A_{k}(z)} \mathfrak{L}^{2 d}(B(\xi ; \alpha t)) \leq \mathfrak{L}^{2 d}(B(z ; \alpha(k+2) t)) \leq q_{1} q_{0}(\alpha(k+2) t)^{2 d-1} .
$$

We conclude that $d_{k} \leq q_{1}(k+2)^{2 d-1}$. Moreover if $\xi_{j}, \xi_{k} \in A_{0}(z)$, then $\rho\left(\xi_{j}, \xi_{k}\right) \leq$ $\rho\left(z, \xi_{j}\right)+\rho\left(z, \xi_{k}\right)<2 \alpha t$ so $\xi_{j}=\xi_{k}$ and $d_{0} \leq 1$.

Since $\Omega \subset B(0, R)$ (see section 1.1) we may assume that $\alpha t \leq R$. In particular

$$
\bigcup_{\xi \in A_{k}(z)} B(\xi ; \alpha t) \subset B(0 ; 2 R)
$$

and we may estimate (see section 1.1)

$$
s q_{0}(\alpha t)^{2 d-1} \leq \sum_{\xi \in A} \mathfrak{L}^{2 d}(B(\xi ; \alpha t)) \leq \mathfrak{L}^{2 d}(B(0 ; 2 R)) \leq q_{1} q_{0} .
$$

Lemma 2.3. If $A \subset \partial \Omega$ is $\alpha$-separated, then for each $\beta>\alpha$ there exists an integer $K=K(\alpha, \beta)$ such that $A$ can be partitioned into $K$ disjoint $\beta t$-separated sets. 
Proof. Let us select from $A$ a maximal $\beta t$-separated subset $A_{1}$. Next from $A \backslash A_{1}$ we select a maximal $\beta t$-separated subset $A_{2}$. We continue this way until we exhaust $A$. Let $A_{s}$ be the last non-empty set in this procedure. Let $\xi \in A_{s}$. Observe that $B(\xi ; \beta t) \cap A_{k} \neq \emptyset$ for $k=1, \ldots, s-1$. In particular $B(\xi ; \beta t)$ contains at least $s$ different elements $\left\{\xi_{1}, \ldots, \xi_{s}\right\}$ from $A$. Since $B\left(\xi_{j} ; \alpha t\right) \cap B\left(\xi_{k} ; \alpha t\right)=\emptyset$ for $j \neq k$ and $B\left(\xi_{j} ; \alpha t\right) \subset B(\xi ;(\beta+\alpha) t)$, then

$$
s q_{0}(\alpha t)^{2 d-1} \leq \sum_{j=1}^{s} \mathfrak{L}^{2 d}\left(B\left(\xi_{j} ; \alpha t\right)\right) \leq \mathfrak{L}^{2 d}(B(\xi ;(\beta+\alpha) t)) \leq q_{1} q_{0}((\beta+\alpha) t)^{2 d-1} .
$$

We can conclude that $s \leq q_{1}\left(\frac{\beta}{\alpha}+1\right)^{2 d-1}$. Now it suffices to choose a natural number $K$ so that $q_{1}\left(\frac{\beta}{\alpha}+1\right)^{2 d-1} \leq K$.

Proposition 2.4. We have the following inequalities for $0<x<1$ :

$$
(1-x)^{\frac{1}{x}}<e^{-1}<(1-x)^{\frac{1}{x}-1} .
$$

Proof. Let $x \in(0,1)$. To prove the left inequality let $f(x):=x+\ln (1-x)$. Since $f^{\prime}(x)=\frac{-x}{1-x}<0$ we have $f(x)<f(0)=0$ and $\frac{1}{x} \ln (1-x)<-1$.

To prove right inequality let $g(x)=\frac{-x}{1-x}-\ln (1-x)$. Since $g^{\prime}(x)=\frac{-x}{(1-x)^{2}}<0$, then $g(x)<g(0)=0$ and $-1<\frac{1-x}{x} \ln (1-x)$.

Now we are ready to state some estimations for polynomials of the form (2).

Lemma 2.5. Let $0<c_{1}<c_{2}$ be constants from Lemma 2.1. For a given a $\in(0,0.5)$ there exist constants $C>2$ and $N_{0} \in \mathbb{N}$ such that for all integers $N \geq N_{0}$, for each $C / \sqrt{c_{1} N}$-separated subset $A$ of $X$ and each integer $m$ with $N \leq m \leq 2 N$ the polynomial $p_{m}(z):=\sum_{\xi \in A}\left\langle z, \nu_{\xi}\right\rangle^{m}$ satisfies

(1) If $z \in \partial \Omega, Q(z):=\left\{\xi \in A: \rho(z, \xi) \geq \frac{C}{2 \sqrt{c_{1} N}}\right\}$, then $\sum_{\xi \in Q(z)}\left|\left\langle z, \nu_{\xi}\right\rangle\right|^{m}<a$.

(2) If $z \in \partial \Omega$, then $Q(z) \backslash A$ has at most one element.

(3) If $\xi_{0} \in A, z \in \partial \Omega$ are such that $\rho\left(z, \xi_{0}\right) \leq \frac{a}{\sqrt{c_{2} N}}$, then

(a) $Q(z)=A \backslash\left\{\xi_{0}\right\}$,

(b) $\left|\left\langle z, \nu_{\xi_{0}}\right\rangle\right|^{m}>1-2 a^{2}$,

(c) $\left|p_{m}(z)\right|>1-2 a^{2}-a$.

(4) $\left|p_{m}(z)\right| \leq \sum_{\xi \in A}\left|\left\langle z, \nu_{\xi}\right\rangle\right|^{m}<1+a$ for all $z \in \partial \Omega$.

Proof. There exists a constant $C>2$ large enough that for $k \in \mathbb{N}_{+}$we have

$$
\sum_{k=1}^{\infty} \frac{q_{1}(k+2)^{2 d-1}}{\exp \left(\frac{k^{2} C^{2}}{4}\right)}<a .
$$

Due to Proposition 2.4 we can estimate:

$$
\lim _{N \rightarrow \infty} \exp \left(\frac{-2 a^{2}}{1-a^{2} N^{-1}}\right)=\exp \left(-2 a^{2}\right)>1-2 a^{2} .
$$

In particular we can choose $N_{0} \in \mathbb{N}$ such that for $N \geq N_{0}$ we have

$$
\exp \left(\frac{-2 a^{2}}{1-a^{2} N^{-1}}\right)>1-2 a^{2} \text {. }
$$


Let $z \in \partial \Omega, Q(z):=\left\{\xi \in A: \rho(z, \xi) \geq \frac{C}{2 \sqrt{c_{1} N}}\right\}$ and

$$
A_{k}(z)=\left\{\xi \in A: \frac{k C}{2 \sqrt{c_{1} N}} \leq \rho(z, \xi)<\frac{(k+1) C}{2 \sqrt{c_{1} N}}\right\} .
$$

Due to Lemma 2.2 the set $A_{0}(z)$ has at most 1 element and

$$
\# A_{k}(z) \leq q_{1}(k+2)^{2 d-1} \text {. }
$$

Since $Q(z) \backslash A=A_{0}(z)$ we have the property (2). Due to Lemma 2.1 for $\xi \in A_{k}(z)$ we have

$$
\left|\left\langle z, \nu_{\xi}\right\rangle\right| \stackrel{\sqrt[3]{3}}{\leq} 1-c_{1} \rho^{2}(z, \xi) \leq 1-\frac{k^{2} C^{2}}{4 N} .
$$

Now we may obtain the property (1):

$$
\begin{aligned}
& \sum_{\xi \in Q(z)}\left|\left\langle z, \nu_{\xi}\right\rangle\right|^{m} \leq \sum_{k=1}^{\infty} \sum_{\xi \in A_{k}(z)}\left|\left\langle z, \nu_{\xi}\right\rangle\right|^{m} \stackrel{100}{\leq} \sum_{k=1}^{\infty} \sum_{\xi \in A_{k}(z)}\left(1-\frac{k^{2} C^{2}}{4 N}\right)^{N} \\
& \stackrel{\text { (6) }}{\leq} \sum_{k=1}^{\infty} \# A_{k}(z) \exp \left(-\frac{k^{2} C^{2}}{4}\right) \stackrel{\text { (g) }}{\leq} \sum_{k=1}^{\infty} \frac{q_{1}(k+2)^{2 d-1}}{\exp \left(\frac{k^{2} C^{2}}{4}\right)} \stackrel{\text { 70 }}{<} a \text {. }
\end{aligned}
$$

Since $A_{0}(z)$ has at most one element we obtain the property (4):

$$
\left|p_{m}(z)\right| \leq \sum_{\xi \in A}\left|\left\langle z, \nu_{\xi}\right\rangle\right|^{m} \leq 1+\sum_{\xi \in Q(z)}\left|\left\langle z, \nu_{\xi}\right\rangle\right|^{m}<1+a .
$$

Now let $\xi_{0} \in A, z \in \partial \Omega$ be such that $\rho\left(z, \xi_{0}\right) \leq \frac{a}{\sqrt{c_{2} N}}<\frac{C}{2 \sqrt{c_{1} N}}$. Since $A_{0}(z)$ has at most 1 element we have $A_{0}(z)=\left\{\xi_{0}\right\}$, which gives the property (3a). Moreover we have:

$$
\left|\left\langle z, \nu_{\xi_{0}}\right\rangle\right| \stackrel{\text { 3. }}{\geq} 1-c_{2} \rho^{2}\left(z, \xi_{0}\right) \geq 1-\frac{a^{2}}{N} .
$$

Now we observe the property (3b) for $N \geq N_{0}$ :

$$
\left|\left\langle z, \nu_{\xi_{0}}\right\rangle\right|^{m} \stackrel{\text { 111 }}{\geq}\left(1-\frac{a^{2}}{N}\right)^{2 N} \stackrel{60}{>} \exp \left(\frac{-a^{2} N^{-1} 2 N}{1-a^{2} N^{-1}}\right) \stackrel{[8}{>} 1-2 a^{2} .
$$

Moreover we may conclude the property (3c):

$$
\left|p_{m}(z)\right| \geq\left|\left\langle z, \nu_{\xi_{0}}\right\rangle\right|^{m}-\sum_{\xi \in Q(z)}\left|\left\langle z, \nu_{\xi}\right\rangle\right|^{m}>1-2 a^{2}-a,
$$

which finishes the proof.

We are ready for main result of this paper.

Theorem 2.6. There exists $K \in \mathbb{N}$ such that for $0<\epsilon<1$ and for each pair of compact, circular and disjoint sets $D, T$ such that $T \subset X, D \subset \partial \Omega$, we can choose $m_{0}=m_{0}(D, T, \epsilon) \in \mathbb{N}$ and a sequence $p_{m}$ of homogeneous polynomials of degree $m$ which satisfy

(1) $\left|p_{m}(z)\right| \leq 2$ for all $z \in \partial \Omega, m>m_{0}$,

(2) $\sum_{k=K m}^{K(m+1)-1}\left|p_{k}(z)\right|^{2} \geq 0.25$ for all $z \in T, m>m_{0}$,

(3) $\sum_{k=K m}^{K(m+1)-1}\left|p_{k}(z)\right|^{2} \leq 2^{-(K m)^{1-\epsilon}}$ for all $z \in D, m>m_{0}$. 
Proof. Let $0<c_{1}<c_{2}$ be from Lemma 2.1. For $a=\frac{1}{4}$ we can choose $C$ from Lemma 2.5. Let $K=K(\alpha, \beta)$ be from Lemma 2.3 for $\alpha=\frac{1}{4 \sqrt{c_{2}}}$ and $\beta=\frac{C}{\sqrt{c_{1}}}$. For $N=K m$ fix a maximal $1 /\left(4 \sqrt{c_{2} N}\right)$-separated subset $A \subset T$. Using Lemma 2.3 we can divide $A$ into at most $K$ disjoint $C / \sqrt{c_{1} N}$-separated subsets $A_{0}, A_{1}, \ldots, A_{K-1}$. We define

$$
p_{K m+j}(z):=\sum_{\xi \in A_{j}}\left\langle z, \nu_{\xi}\right\rangle^{K m+j}
$$

for $j=0,1, \ldots, K-1$. From Lemma 2.5 we infer that there exists $m_{0}$ so high that for $m>m_{0}$ we have $\left|p_{K m+j}(z)\right|<1+a=\frac{5}{4}<2$ for all $z \in \partial \Omega$ and $\left|p_{K m+j}(z)\right|>1-2 a^{2}-a=\frac{6}{16}>0.5$ for

$$
z \in \bigcup_{\xi \in A_{j}} B\left(\xi ; \frac{1}{4 \sqrt{c_{2} N}}\right) \text {. }
$$

Since $A=\bigcup_{j=0}^{K-1} A_{j}$ is a maximal $1 /\left(4 \sqrt{c_{2} N}\right)$-separated subset of $T$ we conclude that

$$
\bigcup_{j=0}^{K-1} \bigcup_{\xi \in A_{j}} B\left(\xi ; \frac{1}{4 \sqrt{c_{2} N}}\right)=\bigcup_{\xi \in A} B\left(\xi ; \frac{1}{4 \sqrt{c_{2} N}}\right) \supset T,
$$

and from this follows that

$$
\sum_{j=K m}^{K(m+1)-1}\left|p_{j}(z)\right|^{2} \geq 0.25 \text { for all } z \in T, m>m_{0} .
$$

Without loss of generality we can assume that $m_{0}$ is so large that $\rho(z, w)>$ $\sqrt{1 / c_{1} N^{\epsilon}}$ for all $z \in D$ and $w \in T$. Due to Lemma 2.2 we have

$$
\# A_{j} \leq q_{1}\left(\frac{\sqrt{c_{1} N}}{C}\right)^{2 d-1} \text {. }
$$

If $\xi \in A$ and $z \in D$, then on the basis of Lemma 2.1 we have

$$
\left|\left\langle z, \nu_{\xi}\right\rangle\right| \leq 1-c_{1} \rho^{2}(z, \xi) \leq 1-\frac{1}{N^{\epsilon}} .
$$

Now for $m_{0}$ large enough, $m>m_{0}, N=K m$ and $z \in D$ we may estimate

$$
\begin{aligned}
\sum_{j=0}^{K-1}\left|p_{K m+j}(z)\right|^{2} & \leq \sum_{j=0}^{K-1} \sum_{\xi \in A_{j}}\left|\left\langle z, \nu_{\xi}\right\rangle\right|^{2 K m+j} \leq \sum_{\xi \in A}\left|\left\langle z, \nu_{\xi}\right\rangle\right|^{N} \\
& \leq \sum_{\xi \in A}\left(1-\frac{1}{N^{\epsilon}}\right)^{N} \leq q_{1} K\left(\frac{\sqrt{c_{1} N}}{C}\right)^{2 d-1}\left(1-\frac{1}{N^{\epsilon}}\right)^{N^{\epsilon} N^{1-\epsilon}} \\
& \leq \frac{1}{2^{N^{1-\epsilon}}}
\end{aligned}
$$

As an application we can present the following result:

Theorem 2.7. Assume that $\Omega$ is a circular, bounded and strictly convex domain with the boundary of class $C^{2}$. Then for any circular subset $E \subset \partial \Omega$ of type $G_{\delta}$ there exists a holomorphic function $f$ which is square integrable on $\Omega \backslash \mathbb{D} E$ and such that $E=E_{\Omega}^{2}(f):=\left\{z \in \partial \Omega: \int_{\mathbb{D} z}|f|^{2} d \mathfrak{L}_{\mathbb{D} z}^{2}=\infty\right\}$. 
Proof. Let $\sigma$ be the natural measure on $\partial \Omega$. On the basis of [8, Theorem 2.6] there exist sequences $\left\{D_{i}\right\}_{i \in \mathbb{N}},\left\{T_{i}\right\}_{i \in \mathbb{N}}$ of compact, circular sets in $\partial \Omega$ such that:

(1) $\bigcup_{i \in \mathbb{N}} D_{i}=\partial \Omega \backslash E$ and $D_{j} \subset D_{j+1}$ for $j \in \mathbb{N}$,

(2) $T_{j} \cap D_{j}=\emptyset$ for $j \in \mathbb{N}$,

(3) $E=\bigcap_{j=1}^{\infty} \bigcup_{i=j}^{\infty} T_{i}$,

(4) $\sigma\left(\partial \Omega \backslash\left(E \cup D_{j}\right)\right) \leq 2^{-j}$.

Since $\Omega$ is a strictly convex domain then $X=\partial \Omega$. Let $K$ be a natural number from Theorem 2.6. We can use Theorem 2.6 once again and conclude that there exist a sequence of natural numbers $\left\{m_{j}\right\}_{j \in \mathbb{N}}$ and a sequence of homogeneous polynomials $\left\{p_{m}\right\}_{m \in \mathbb{N}}$ such that

(1) $m_{j}<m_{j+1}$ for $j \in \mathbb{N}$,

(2) $\left|p_{m}(z)\right| \leq 2$ for all $z \in \partial \Omega, m>m_{0}$,

(3) $\sum_{k=K m_{j}}^{K\left(m_{j}+1\right)-1}\left|p_{k}(z)\right|^{2} \geq 0.25$ for all $z \in T_{j}$,

(4) $\sum_{k=K m_{j}}^{K\left(m_{j}+1\right)-1}\left|p_{k}(z)\right|^{2} \leq 2^{-j}$ for all $z \in D_{j}$.

Now we can define

$$
f=\frac{1}{\sqrt{\pi}} \sum_{j=1}^{\infty} \sum_{k=K m_{j}}^{K\left(m_{j}+1\right)-1} \sqrt{k+1} p_{k} .
$$

Observe that for $z \in \partial \Omega$ we have

$$
\int_{\mathbb{D} z}|f|^{2} d \mathfrak{L}_{\mathbb{D} z}^{2}=\sum_{j=1}^{\infty} \sum_{k=K m_{j}}^{K\left(m_{j}+1\right)-1}\left|p_{k}(z)\right|^{2} .
$$

In particular for $z \in E$ we have

$$
\int_{\mathbb{D} z}|f|^{2} d \mathfrak{L}_{\mathbb{D} z}^{2} \geq \sum_{j: z \in T_{j}} 0.25=\infty .
$$

If $z \in \partial \Omega \backslash E$, then there exists $j_{0}$ such that $z \in D_{j}$ for $j \geq j_{0}$. In particular

$$
\int_{\mathbb{D} z}|f|^{2} d \mathfrak{L}_{\mathbb{D} z}^{2} \leq \sum_{j=1}^{j_{0}-1} \sum_{k=K m_{j}}^{K\left(m_{j}+1\right)-1}\left|p_{k}(z)\right|^{2}+\sum_{j=j_{0}}^{\infty} 2^{-j}<\infty .
$$

Now we prove that $f$ is square integrable on $\Omega \backslash \mathbb{D} E$. There exists $M>0$ such that $\int_{\Omega \backslash \mathbb{D E} E}|f|^{2} d \mathfrak{L}^{2 d} \leq M \int_{\partial \Omega \backslash E} \int_{\mathbb{D} z}|f|^{2} d \mathfrak{L}_{\mathbb{D} z}^{2} d \sigma(z)$. In particular we may estimate

$$
\begin{aligned}
\int_{\Omega \backslash \mathbb{D E}}|f|^{2} d \mathfrak{L}^{2 d} & \leq M \sum_{j=1}^{\infty} \int_{\partial \Omega \backslash E} \sum_{k=K m_{j}}^{K\left(m_{j}+1\right)-1}\left|p_{k}\right|^{2} d \sigma \\
& \leq M \sum_{j=1}^{\infty} 2^{-j} \sigma\left(D_{j}\right)+M \sum_{j=1}^{\infty} 4 K \sigma\left(\partial \Omega \backslash\left(E \cup D_{j}\right)\right) \\
& \leq M \sigma(\partial \Omega)+4 K M<\infty .
\end{aligned}
$$

\section{REFERENCES}

[1] J. Globevnik, E. L. Stout, Highly noncontinuable functions on convex domains, Bull. Sci. Math. 104 (1980), 417-439. MR602409 (82c:32013)

[2] J. Globevnik, E. L. Stout, Holomorphic functions with highly noncontinuable boundary behavior, J. d'Analyse Math. 41 (1982), 211-216. MR687952(84h:32016) 
[3] J. Globevink, Holomorphic functions which are highly nonintegrable at the boundary, Israel J. Math. 115 (2000), 195-203. MR1749678 (2001c:32005)

[4] P. Jakóbczak, Description of exceptional sets in the circles for functions from the Bergman space, Czechoslovak Journal of Mathematics no. 47, (1997), 633-649. MR1479310|(98h:32042)

[5] P. Jakóbczak, Highly non-integrable functions in the unit ball. Israel J. Math 97 (1997), 175-181. MR1441246(98b:32002)

[6] P. Jakóbczak, Exceptional sets of slices for functions from the Bergman Space in the ball, Canad. Math. Bull. 44(2), (2001), 150-159 MR1827853 (2002c:32007)

[7] P. Kot, Description of simple exceptional sets in the unit ball, Czechoslovak Mathematical Journal 54 (129) (2004), 55-63. MR2040218 (2004k:32008)

[8] P. Kot, Maximum sets of semicontinuous functions, Potential Analysis (2005) 23, 323-356. MR2139570 (2005m:31004)

[9] S. G. Krantz, Function theory of several complex variables, PWN Warsaw 1991. MR:1162310 (93c:32001)

[10] J. Ryll and P. Wojtaszczyk, On homogeneous polynomials on a complex ball, Trans. Amer. Math. Soc. 276 (1983), 107-116. MR684495 (84f:32004)

[11] P. Wojtaszczyk, On values of homogeneous polynomials in discrete sets of points, Studia Math. 84 (1986), 97-104. MR871849 (88e:32014)

[12] P. Wojtaszczyk, On highly nonintegrable functions and homogeneous polynomials, Annales Polonici Mathematici no. 65, (1997), 245-251. MR1441179 (98a:32007)

Politechnika Krakowska, Instytut Matematyki, Ul. Warszawska 24, 31-155 Kraków, POLAND

E-mail address: pkot@usk.pk.edu.pl 